Published in final edited form as:

J Investig Med. 2011 August ; 59(6): 881-886. doi:10.231/JIM.0b013e31821b8755.

\title{
Vitamin D and the Immune System
}

\author{
Cynthia Aranow, MD[Investigator] \\ Feinstein Institute for Medical Research, Manhasset, N.Y.
}

\section{Abstract}

It is now clear that vitamin D has important roles in addition to its classic effects on calcium and bone homeostasis. As the vitamin $\mathrm{D}$ receptor is expressed on immune cells (B cells, T cells and antigen presenting cells) and these immunologic cells are all are capable of synthesizing the active vitamin D metabolite, vitamin $\mathrm{D}$ has the capability of acting in an autocrine manner in a local immunologic milieu. Vitamin D can modulate the innate and adaptive immune responses. Deficiency in vitamin D is associated with increased autoimmunity as well as an increased susceptibility to infection. As immune cells in autoimmune diseases are responsive to the ameliorative effects of vitamin D, the beneficial effects of supplementing vitamin D deficient individuals with autoimmune disease may extend beyond the effects on bone and calcium homeostasis.

The immune system defends the body from foreign, invading organisms, promoting protective immunity while maintaining tolerance to self. The implications of vitamin D deficiency on the immune system have become clearer in recent years and in the context of vitamin D deficiency, there appears to be an increased susceptibility to infection and a diathesis, in a genetically susceptible host to autoimmunity.

The classical actions of vitamin $\mathrm{D}$ are to promote calcium homeostasis and to promote bone health. Vitamin D enhances absorption of calcium in the small intestine and stimulates osteoclast differentiation and calcium reabsorption of bone. Vitamin D additionally promotes mineralization of the collagen matrix in bone. In humans, vitamin D is obtained from the diet or it is synthesized it in the skin (reviewed in [1]). As vitamin D is cutaneously produced after exposure to UV B light, its synthesis is influenced by latitude, season, use of sunblock and skin pigmentation. Melanin absorbs UVB radiation inhibiting the synthesis of vitamin D from 7-dihydrocholesterol. This initial vitamin D compound is inactive and it is next hydroxylated in the liver to form $25 \mathrm{OH}$ vitamin D3 (25 D). $25 \mathrm{D}$ is also an inactive compound, but is the most reliable measurement of an individual's vitamin D status. It is converted in the kidney to the active compound 1,25 dihydroxy vitamin $\mathrm{D}(1,25 \mathrm{D})$ or calcidiol by 1- $\alpha$-hydroxylase (CYP27B1), an enzyme which is stimulated by PTH . 1,25 D may be further metabolized to the inactive 1,24,25 vitamin D by 24-hydroxylase (CYP24). $1,25 \mathrm{D}$ levels are tightly regulated in a negative feedback loop. 1,25 D both inhibits renal 1$\alpha$-hydroxylase and stimulates the 24-hydroxylase enzymes, thus maintaining circulating levels within limited boundaries and preventing excessive vitamin D activity/signaling.

Correspondence: Cynthia Aranow 350 Community Drive Manhasset, NY 11030516 562-3837 $516562-2537$ (fax) caranow@nshs.edu .

Address for reprints: same as corresponding author

This is a PDF file of an unedited manuscript that has been accepted for publication. As a service to our customers we are providing this early version of the manuscript. The manuscript will undergo copyediting, typesetting, and review of the resulting proof before it is published in its final citable form. Please note that during the production process errors may be discovered which could affect the content, and all legal disclaimers that apply to the journal pertain. 
$1,25 \mathrm{D}$ acts on the intestine where it stimulates calcium reabsorption, and upon bone, where it promotes osteoblast differentiation and matrix calcification. The active hormone exerts its effects on these tissues by binding to the vitamin $\mathrm{D}$ receptor (VDR). This complex dimerizes with the retinoid X receptor (RXR) and the 1,25D-VDR-RXR heterodimer translocates to the nucleus where it binds vitamin D responsive elements (VDRE) in the promoter regions of vitamin D responsive genes and induces expression of these vitamin D responsive genes.

Many tissues other than the skeletal and intestine express the VDR including cells in the bone marrow, brain, colon, breast and malignant cells and immune cells suggesting that vitamin D may have functions other than calcium and bone homeostasis[2]. Additionally, tissues other than the kidney express 1- $\alpha$-hydroxylase and are capable of converting $25 \mathrm{D}$ to $1,25 \mathrm{D}$, in non-renal compartments[1, 3-4]. Therefore, in addition to its endocrine functions, vitamin D may act in a paracrine or autocrine manner. Some of the more recently recognized non-classical actions of vitamin D include effects upon cell proliferation and differentiation as well immunologic effects resulting in an ability to maintain tolerance and to promote protective immunity. As antigen presenting cells (macrophages and dendritic cells), T cells and $\mathrm{B}$ cells have the necessary machinery to synthesize and respond to $1,25 \mathrm{D}$, vitamin $\mathrm{D}$ may act in a paracrine or autocrine manner in an immune environment. Moreover, local levels of 1,25 D may differ from systemic, circulating levels as local regulation of the enzymes synthesizing and inactivating vitamin $\mathrm{D}$ are different from the controls originating in the kidney. The extrarenal 1- $\alpha$-hydroxylase enzyme in macrophages differs from the renal hydroxylase as it is not regulated by PTH[5]. Instead, it is dependent upon circulating levels of $25 \mathrm{D}$ or it may be induced by cytokines such as IFN- $\gamma, \mathrm{IL}-1$ or TNF- $\alpha$ [6]. Furthermore, the macrophage 24 hydroxylase enzyme is a non-functional splice variant, so there is no negative feedback of local 1,25 D production by $1,25 \mathrm{D}$.

\section{Vitamin D and Protective Immunity}

Vitamin D has been used (unknowingly) to treat infections such as tuberculosis before the advent of effective antibiotics. Tuberculosis patients were sent to sanatoriums where treatment included exposure to sunlight which was thought to directly kill the tuberculosis. Cod liver oil, a rich source of vitamin D has also been employed as a treatment for tuberculosis as well as for general increased protection from infections[7].

There have been multiple cross-sectional studies associating lower levels of vitamin D with increased infection. One report studied almost 19,000 subjects between 1988 and 1994. Individuals with lower vitamin D levels $(<30 \mathrm{ng} / \mathrm{ml})$ were more likely to self-report a recent upper respiratory tract infection than those with sufficient levels, even after adjusting for variables including season, age, gender, body mass and race[8]. Vitamin D levels fluctuate over the year. Although rates of seasonal infections varied, and were lowest in the summer and highest in the winter, the association of lower serum vitamin D levels and infection held during each season. Another cross-sectional study of 800 military recruits in Finland stratified men by serum vitamin D levels[9]. Those recruits with lower vitamin D levels lost significantly more days from active duty secondary to upper respiratory infections than recruits with higher vitamin $D$ levels (above 40nmol). There have been a number of other cross-sectional studies looking at vitamin D levels and rates of influenza [10] as well as other infections including bacterial vaginosis[11] and HIV[12-13]. All have reported an association of lower vitamin D levels and increased rates of infection.

Results of studies looking at potential benefits of administering vitamin D to decrease infection have not been consistent, most likely secondary to a number of methodologic concerns[14]. One recent well-designed prospective, double blind placebo study using an objective outcome, nasopharyngeal swab culture (and not self report), and a therapeutic dose 
of vitamin D showed that vitamin D administration resulted in a statistically significant $(42 \%)$ decrease in the incidence of influenza infection[15].

The beneficial effects of vitamin $\mathrm{D}$ on protective immunity are due in part to its effects on the innate immune system. It is known that macrophages recognize lipopolysacharide LPS, a surrogate for bacterial infection, through toll like receptors (TLR). Engagement of TLRs leads to a cascade of events that produce peptides with potent bacterialcidal activity such as cathelocidin and beta defensin 4[16]. These peptides colocalize within phagosomes with injested bacteria where they disrupt bacterial cell membranes and have potent antimicrobacterial activity [17].

Vitamin D plays an important part in the innate antimicrobial response. TLR binding leads to increased expression of both the 1- $\alpha$-hydroxylase and the VDR[17-18]. This results in binding of the 1,25 D-VDR-RXR heterodimer to the VDREs of the genes for cathelocidin and beta defensin 4 and subsequent transcription of these proteins. Transcription of cathelocidin is absolutely dependent on sufficient $25 \mathrm{D}$ [17]. It is now clear that transcription of beta defensin 4 requires binding of NFkB to appropriate response elements on the beta defensin 4 RNA[19]. TLR 2-1 signaling facilitates IL-1 receptor engagement which results in translocation of NFkB to its binding site[19].

\section{Vitamin D and Autoimmune Disease}

There is increasing epidemiologic evidence linking vitamin D deficiency and autoimmune diseases including multiple sclerosis (MS), rheumatoid arthritis (RA), diabetes mellitus (DM), inflammatory bowel disease and systemic lupus erythematosus (SLE) (reviewed in reference[20]. Reports of low serum vitamin D predicting development of autoimmune disease in the future have been published for MS, autoimmune DM and RA[21-23]. There is also data linking decreased in utero exposure to vitamin D and islet cell autoimmunity[24]. Lower in utero exposure assessed by a lower maternal intake of vitamin D during pregnancy in women whose prospective child was at risk of developing autoimmune DM is associated with a statistically increased risk of the child developing pancreatic autoimmunity.

Vitamin D has also been shown to facilitate progression of existing autoimmune disease. In one study, 161 patients with an early undifferentiated connective tissue disease were followed for a mean of over 2 years[25]. Most patients did not progress and remained in an undifferentiated state. Thirty-five (21\%) patients went on to develop a defined rheumatologic diagnosis including RA, SLE, Mixed Connective Tissue Disease, and Sjogren's Disease while 126 did not progress. Baseline characteristics of the two groups were similar. Importantly, the mean vitamin D level was significantly lower in the group that progressed to a definitive disease.

There have been many studies of vitamin D status in lupus patients from across the globe (reviewed in [26]). Vitamin D levels are typically lower in patients than in disease or normal controls. Deficiency of vitamin D is extremely common, often with more than $50 \%$ of lupus patients with deficient levels and severe deficiency (vitamin D levels less than $10 \mathrm{ng} / \mathrm{ml}$ ) is not uncommon. Disease activity has been shown to correlate inversely with vitamin D in many but not all studies. Similar correlations between low levels of vitamin D and disease activity and severity have been observed in other autoimmune diseases such as MS and RA[27-30].

\section{Vitamin D and Immunologic Function}

Vitamin D has numerous effects on cells within the immune system. It inhibits B cell proliferation and blocks B cell differentiation and immunoglobulin secretion[31-32]. 
Vitamin D additionally suppresses T cell proliferation[33] and results in a shift from a Th1 to a Th2 phenotype[34-35]. Furthermore, it affects T cell maturation with a skewing away from the inflammatory Th17 phenotype[36-37] and facilitates the induction of T regulatory cells [38-41]. These effects result in decreased production of inflammatory cytokines (IL-17, IL-21) with increased production of anti-inflammatory cytokines such as IL-10 (Figure 1A). Vitamin D also has effects on monocytes and dendritic cells (DCs). It inhibits monocyte production of inflammatory cytokines such as IL-1, IL-6, IL-8, IL-12 and TNF $\alpha$ [42]. It additionally inhibits DC differentiation and maturation with preservation of an immature phenotype as evidenced by a decreased expression of MHC class II molecules, costimulatory molecules and IL12[43-45] (Figure 1B).

Inhibition of DC differentiation and maturation is particularly important in the context of autoimmunity and the abrogation of self tolerance. Antigen presentation to a $\mathrm{T}$ cell by a mature DC facilitates an immune response against that antigen while antigen presentation by an immature DC facilitates tolerance. Self-antigens are abundant in the normal state from physiologic cell death and turnover. However, presentation of these self-antigens is usually by immature DCs so that tolerance to self is maintained.

Given the importance of vitamin D for a functional immune system and the profound deficiency observed in autoimmune disease, as well as the correlation of deficiency with more active disease, an important issue is whether or not the immune components in autoimmune disease are capable of responding appropriately to vitamin D. Immune cells (B cells, T cells, monocytes, DCs) from multiple autoimmune diseases appear to respond to the immunomodulatory effects of vitamin D. Examples of vitamin D responsiveness by immunologic components in different autoimmune disease follow: B cells: Abnormalities of B cells from lupus patients may be partially reversed by vitamin D. Both spontaneous and stimulated immunoglobulin production from B cells from active lupus patients are significantly decreased by pre-incubating cells with 1,25 vitamin D[46]. Additionally, preincubation with vitamin D significantly decreases spontaneous production of anti-DNA antibodies by approximately 60\%[46]. T cells: T cells from patients with MS respond to vitamin $\mathrm{D}$. The proliferation of stimulated CD4 cells from MS patients and controls are similarly inhibited after preincubation in increasing concentrations of vitamin D[27]. Moreover, Th17 polarized T cells from both controls and MS patients respond when incubated with vitamin D; both are downregulated with diminished production of IL-17 and gamma interferon[27]. Monocytes: Vitamin D inhibits the production of inflammatory cytokines (IL-1, TNF $\alpha$ ) by monocyes. Cytokine production by monocytes from both normal controls and from patients with autoimmune diabetes (type 1 or latent autoimmune diabetics) is significantly diminished by vitamin D[47]. TLR 4 stimulation by LPS or LTA (leipoteichoic acid) is similarly inhibited by exposure to vitamin D[47]. DCs: Lupus DCs are susceptible to the effects of vitamin D. LPS induced DC maturation is inhibited by preincubation with vitamin D resulting in suppressed expression of HLA class II and costimulatory molecules. The response of lupus cells to LPS stimulation is similarly suppressed by vitamin D[48]. Furthermore, vitamin D affects the expression of the interferon (IFN) signature in SLE. Interferon is produced by plasmacytoid DCs; the IFN signature refers to the overexpression of IFN $\alpha$ inducible genes in peripheral blood mononuclear cells (PBMC s) of lupus patients[49]. The signature occurs in approximately $50 \%$ of patients and correlates with disease activity[50-52]. We have observed that interferon inducible genes are overexpressed in lupus patients with low serum vitamin $\mathrm{D}$ compared to normal serum vitamin D (Figure 2A). Expression of these interferon inducible genes may be diminished in lupus patients after receiving vitamin $\mathrm{D}$ supplementation (Figure 2B). In fact, we have observed that an IFN signature response, the decrease in expression of IFN inducible genes is 2.1 times more likely to occur in vitamin D supplemented lupus patients (unpublished data Ben-Zvi, I). There is currently a double-blind 
placebo controlled NIH sponsored trial (ClinicalTrials.gov identifier: NCT00710021) assessing the potential ability of vitamin $\mathrm{D}$ to suppress the interferon signature in patients with SLE.

\section{Conclusions}

Vitamin D has important functions beyond those of calcium and bone homeostasis which include modulation of the innate and adaptive immune responses. Vitamin D deficiency is prevalent in autoimmune disease. Cells of the immune system are capable of synthesizing and responding to vitamin D. Immune cells in autoimmune diseases are responsive to the ameliorative effects of vitamin D suggesting that the beneficial effects of supplementing vitamin $\mathrm{D}$ deficient individuals with autoimmune disease may extend beyond effects on bone and calcium homeostasis.

\section{References}

1. Holick MF. Vitamin D deficiency. N Engl J Med. 2007; 357(3):266-81. [PubMed: 17634462]

2. Bikle D. Nonclassic actions of vitamin D. J Clin Endocrinol Metab. 2009; 94(1):26-34. [PubMed: 18854395]

3. Townsend K, et al. Biological actions of extra-renal 25-hydroxyvitamin D-1alpha-hydroxylase and implications for chemoprevention and treatment. J Steroid Biochem Mol Biol. 2005; 97(1-2):103-9. [PubMed: 16081283]

4. Nagpal S, Na S, Rathnachalam R. Noncalcemic actions of vitamin D receptor ligands. Endocr Rev. 2005; 26(5):662-87. [PubMed: 15798098]

5. Wu S, et al. Splice variants of the CYP27b1 gene and the regulation of 1,25-dihydroxyvitamin D3 production. Endocrinology. 2007; 148(7):3410-8. [PubMed: 17395703]

6. van Etten E, et al. Regulation of vitamin D homeostasis: implications for the immune system. Nutr Rev. 2008; 66(10 Suppl 2):S125-34. [PubMed: 18844839]

7. Williams C. On the use and administration of cod-liver oil in pulmonary consumption. London Journal of Medicine. 1849; 1:1-18.

8. Ginde AA, Mansbach JM, Camargo CA Jr. Association between serum 25-hydroxyvitamin D level and upper respiratory tract infection in the Third National Health and Nutrition Examination Survey. Arch Intern Med. 2009; 169(4):384-90. [PubMed: 19237723]

9. Laaksi I, et al. An association of serum vitamin D concentrations $<40 \mathrm{nmol} / \mathrm{L}$ with acute respiratory tract infection in young Finnish men. Am J Clin Nutr. 2007; 86(3):714-7. [PubMed: 17823437]

10. Cannell JJ, et al. Epidemic influenza and vitamin D. Epidemiol Infect. 2006; 134(6):1129-40. [PubMed: 16959053]

11. Bodnar LM, Krohn MA, Simhan HN. Maternal vitamin D deficiency is associated with bacterial vaginosis in the first trimester of pregnancy. J Nutr. 2009; 139(6):1157-61. [PubMed: 19357214]

12. Villamor E. A potential role for vitamin D on HIV infection? Nutr Rev. 2006; 64(5 Pt 1):226-33. [PubMed: 16770943]

13. Rodriguez M, et al. High frequency of vitamin D deficiency in ambulatory HIV-Positive patients. AIDS Res Hum Retroviruses. 2009; 25(1):9-14. [PubMed: 19108690]

14. Yamshchikov AV, et al. Vitamin D for treatment and prevention of infectious diseases: a systematic review of randomized controlled trials. Endocr Pract. 2009; 15(5):438-49. [PubMed: 19491064]

15. Urashima M, et al. Randomized trial of vitamin D supplementation to prevent seasonal influenza A in schoolchildren. Am J Clin Nutr. 2010; 91(5):1255-60. [PubMed: 20219962]

16. Gallo RL, et al. Biology and clinical relevance of naturally occurring antimicrobial peptides. J Allergy Clin Immunol. 2002; 110(6):823-31. [PubMed: 12464945]

17. Liu PT, et al. Toll-like receptor triggering of a vitamin D-mediated human antimicrobial response. Science. 2006; 311(5768):1770-3. [PubMed: 16497887] 
18. Wang TT, et al. Cutting edge: 1,25-dihydroxyvitamin D3 is a direct inducer of antimicrobial peptide gene expression. J Immunol. 2004; 173(5):2909-12. [PubMed: 15322146]

19. Liu PT, et al. Convergence of IL-1beta and VDR activation pathways in human TLR2/1-induced antimicrobial responses. PLoS One. 2009; 4(6):e5810. [PubMed: 19503839]

20. Adorini L. Intervention in autoimmunity: the potential of vitamin D receptor agonists. Cell Immunol. 2005; 233(2):115-24. [PubMed: 15936743]

21. Munger KL, et al. Serum 25-hydroxyvitamin D levels and risk of multiple sclerosis. JAMA. 2006; 296(23):2832-8. [PubMed: 17179460]

22. Littorin B, et al. Lower levels of plasma 25-hydroxyvitamin D among young adults at diagnosis of autoimmune type 1 diabetes compared with control subjects: results from the nationwide Diabetes Incidence Study in Sweden (DISS). Diabetologia. 2006; 49(12):2847-52. [PubMed: 17072585]

23. Merlino LA, et al. Vitamin D intake is inversely associated with rheumatoid arthritis: results from the Iowa Women's Health Study. Arthritis Rheum. 2004; 50(1):72-7. [PubMed: 14730601]

24. Fronczak CM, et al. In utero dietary exposures and risk of islet autoimmunity in children. Diabetes Care. 2003; 26(12):3237-42. [PubMed: 14633808]

25. Zold E, et al. Vitamin D deficiency in undifferentiated connective tissue disease. Arthritis Res Ther. 2008; 10(5):R123. [PubMed: 18928561]

26. Kamen D, Aranow C. Vitamin D in systemic lupus erythematosus. Curr Opin Rheumatol. 2008; 20(5):532-7. [PubMed: 18698173]

27. Correale J, Ysrraelit MC, Gaitan MI. Immunomodulatory effects of Vitamin D in multiple sclerosis. Brain. 2009; 132(Pt 5):1146-60. [PubMed: 19321461]

28. Smolders J, et al. Association of vitamin D metabolite levels with relapse rate and disability in multiple sclerosis. Mult Scler. 2008; 14(9):1220-4. [PubMed: 18653736]

29. Craig SM, et al. Vitamin D status and its associations with disease activity and severity in African Americans with recent-onset rheumatoid arthritis. J Rheumatol. 2010; 37(2):275-81. [PubMed: 20032100]

30. Patel S, et al. Association between serum vitamin D metabolite levels and disease activity in patients with early inflammatory polyarthritis. Arthritis Rheum. 2007; 56(7):2143-9. [PubMed: 17599737]

31. Lemire JM, et al. 1 alpha,25-dihydroxyvitamin D3 suppresses proliferation and immunoglobulin production by normal human peripheral blood mononuclear cells. J Clin Invest. 1984; 74(2):65761. [PubMed: 6611355]

32. Chen S, et al. Modulatory effects of 1,25-dihydroxyvitamin D3 on human B cell differentiation. J Immunol. 2007; 179(3):1634-47. [PubMed: 17641030]

33. Bhalla AK, et al. 1,25-Dihydroxyvitamin D3 inhibits antigen-induced T cell activation. J Immunol. 1984; 133(4):1748-54. [PubMed: 6206136]

34. Mattner F, et al. Inhibition of Th1 development and treatment of chronic-relapsing experimental allergic encephalomyelitis by a non-hypercalcemic analogue of 1,25-dihydroxyvitamin $\mathrm{D}(3)$. Eur J Immunol. 2000; 30(2):498-508. [PubMed: 10671205]

35. Boonstra A, et al. 1alpha,25-Dihydroxyvitamin d3 has a direct effect on naive CD4(+) T cells to enhance the development of Th2 cells. J Immunol. 2001; 167(9):4974-80. [PubMed: 11673504]

36. Tang J, et al. Calcitriol suppresses antiretinal autoimmunity through inhibitory effects on the Th17 effector response. J Immunol. 2009; 182(8):4624-32. [PubMed: 19342637]

37. Daniel C, et al. Immune modulatory treatment of trinitrobenzene sulfonic acid colitis with calcitriol is associated with a change of a T helper (Th) $1 / \mathrm{Th} 17$ to a Th2 and regulatory T cell profile. $\mathrm{J}$ Pharmacol Exp Ther. 2008; 324(1):23-33. [PubMed: 17911375]

38. Gregori S, et al. Regulatory T cells induced by 1 alpha,25-dihydroxyvitamin D3 and mycophenolate mofetil treatment mediate transplantation tolerance. J Immunol. 2001; 167(4): 1945-53. [PubMed: 11489974]

39. Barrat FJ, et al. In vitro generation of interleukin 10-producing regulatory CD4(+) T cells is induced by immunosuppressive drugs and inhibited by T helper type 1 (Th1)- and Th2-inducing cytokines. J Exp Med. 2002; 195(5):603-16. [PubMed: 11877483] 
40. Gorman S, et al. Topically applied 1,25-dihydroxyvitamin D3 enhances the suppressive activity of CD4+CD25+ cells in the draining lymph nodes. J Immunol. 2007; 179(9):6273-83. [PubMed: 17947703]

41. Penna G, et al. Expression of the inhibitory receptor ILT3 on dendritic cells is dispensable for induction of CD4+Foxp3+ regulatory T cells by 1,25-dihydroxyvitamin D3. Blood. 2005; 106(10): 3490-7. [PubMed: 16030186]

42. Almerighi C, et al. 1Alpha,25-dihydroxyvitamin D3 inhibits CD40L-induced pro-inflammatory and immunomodulatory activity in human monocytes. Cytokine. 2009; 45(3):190-7. [PubMed: 19186073]

43. Piemonti L, et al. Vitamin D3 affects differentiation, maturation, and function of human monocytederived dendritic cells. J Immunol. 2000; 164(9):4443-51. [PubMed: 10779743]

44. Griffin MD, et al. Dendritic cell modulation by 1alpha,25 dihydroxyvitamin D3 and its analogs: a vitamin D receptor-dependent pathway that promotes a persistent state of immaturity in vitro and in vivo. Proc Natl Acad Sci U S A. 2001; 98(12):6800-5. [PubMed: 11371626]

45. Szeles L, et al. 1,25-dihydroxyvitamin D3 is an autonomous regulator of the transcriptional changes leading to a tolerogenic dendritic cell phenotype. J Immunol. 2009; 182(4):2074-83. [PubMed: 19201860]

46. Linker-Israeli M, et al. Vitamin D(3) and its synthetic analogs inhibit the spontaneous in vitro immunoglobulin production by SLE-derived PBMC. Clin Immunol. 2001; 99(1):82-93. [PubMed: 11286544]

47. Du T, et al. Modulation of monocyte hyperresponsiveness to TLR ligands by 1,25-dihydroxyvitamin D3 from LADA and T2DM. Diabetes Res Clin Pract. 2009; 83(2):208-14. [PubMed: 19010563]

48. Ben-Zvi I, et al. The impact of vitamin D on dendritic cell function in patients with systemic lupus erythematosus. PLoS One. 2010; 5(2):e9193. [PubMed: 20169063]

49. Bennett L, et al. Interferon and granulopoiesis signatures in systemic lupus erythematosus blood. J Exp Med. 2003; 197(6):711-23. [PubMed: 12642603]

50. Baechler EC, et al. Interferon-inducible gene expression signature in peripheral blood cells of patients with severe lupus. Proc Natl Acad Sci U S A. 2003; 100(5):2610-5. [PubMed: 12604793]

51. Kirou KA, et al. Activation of the interferon-alpha pathway identifies a subgroup of systemic lupus erythematosus patients with distinct serologic features and active disease. Arthritis Rheum. 2005; 52(5):1491-503. [PubMed: 15880830]

52. Feng X, et al. Association of increased interferon-inducible gene expression with disease activity and lupus nephritis in patients with systemic lupus erythematosus. Arthritis Rheum. 2006; 54(9): 2951-62. [PubMed: 16947629] 


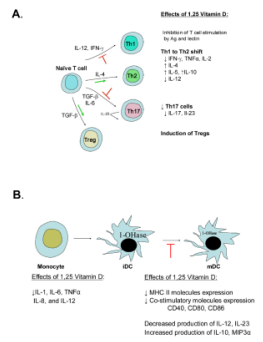

Figure 1.

A. Effects of 1,25 Vitamin D on T cells include suppression of T cell proliferation, a shift from Th1 to a Th2 development, inhibition of Th17 cell development and facilitation of T regulatory cells. B. Effects of 1,25 Vitamin D on monocytes and dendric cells include inhibition of inflammatory cytokine production by monocytes and inhibition of dendritic cell differentiation and maturation. 
A.
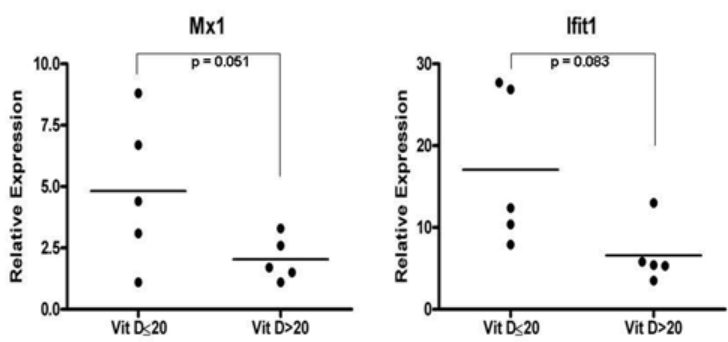

B.

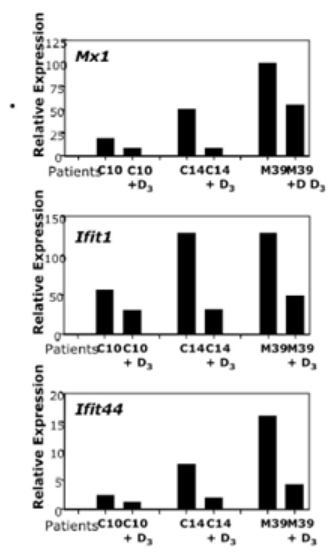

Figure 2.

A. Relative expression of 2 IFN $\alpha$ inducible genes, Mx1 and Ifit1 in SLE patients with vitamin D deficiency $(\leq 20 \mathrm{ng} / \mathrm{ml})$ and sufficiency $(>20 \mathrm{ng} / \mathrm{ml})$. Relative expression of these genes was determined by RTPCR on PBMCs from clinically stable SLE patients.

Expression of interferon inducible genes is higher in patients with SLE with low serum vitamin D (unpublished data Ben-Zvi, I). B. Relative expression of 3 IFN $\alpha$ inducible genes (Mx1, Ifi1 and Ifit44) before and after $\left(+\mathrm{D}_{3}\right)$ supplementation with vitamin $\mathrm{D}_{3}$ in 3 SLE patients. Vitamin D supplementation reduces expression of IFN $\alpha$ inducible genes (unpublished data Ben-Zvi, I). 